Background Sport can represent an important aspect of peoples' lives, for professional and recreational athletes. Some sports involve a high risk of orofacial trauma, particularly contact sports. Undoubtedly, mouthguards are considered by many as an effective solution in preventing injury.

Objective This systematic review aimed to measure the effectiveness of mouthguards in preventing oral-facial injuries.

Design Preferred Reporting Items for Systematic Reviews (PRISMA)

Setting Available electronic databases

Patients (or Participants) Contact sport athletes

Interventions (or Assessment of Risk Factors) MEDLINE and EMBASE electronic databases searches were supplemented by manual searching, of in-vivo studies examining prevention of orofacial injuries by protective devices that cover the teeth and surrounding soft tissues (mouthguards).

Main Outcome Measurements Included studies were assessed for methodological quality and bias. A meta-analysis was performed on data from selected studies, comparing the number of injuries for mouthguard users and non-users. Results are presented as forest plots and relative risks between different studies compared.

Results 1745 records were identified. Fifteen studies were eligible for inclusion in the qualitative synthesis, seven were included in the meta-analysis. The overall quality of studies was low, primarily due to a high degree of bias. The degree of heterogeneity was high throughout the studies affecting most of the variables such as sport, athletes' age, the definition of injury used and type of mouthguard. The meta-analysis showed that wearing a mouthguard was associated with a greater risk of orofacial injury when compared to non-users (overall RR 0.85\%, 95\% CI 0.78-0.93). However, the high degree of heterogeneity questions the validity of the summary estimate. $(\mathrm{I}$-squared $=91.2 \%$ ).

Conclusions There is insufficient evidence to support or refute the use of mouthguards to prevent orofacial injuries. Further research from high quality prospective cohort studies are needed to investigate the protective nature of mouthguards from orofacial injuries, when used in sports. The meta-analysis findings could be explained by athletes being more physical in the knowledge that they are protected.

\section{CAN A BEHAVIOUR CHANGE INTERVENTION IMPROVE ATHLETE ORAL HEALTH?}

Julie Gallagher, Paul Ashley, lan Needleman. UCL Eastman Dental Institute, London, UK

10.1136/bjsports-2021-IOC.313

Background Poor oral health of elite athletes is associated with negative performance impacts. There is a need for oral health promotion and prevention strategies that are effective within the elite sport environment

Objective To develop, implement and evaluate a pragmatic oral health promotion intervention based on contemporary behaviour change theory and informed by input from all stakeholders in elite sport.

Design Interrupted time-series intervention study.

Setting Three separate UK elite athlete-training centres.

Patients (or Participants) 62 athletes from two Olympic and one professional athlete training squads; 55 athletes completed the study.
Interventions (or Assessment of Risk Factors) Two levels: Level 1. Athletes and support team viewed combined one 10-minute presentation focussed on building motivation and three 90 -second information films. Level 2. Athletes alone received oral health screening, personalised advice and an oral health toolkit focussed on opportunity and capability. Follow-up of athletes at one and three months.

Main Outcome Measurements Impact on performance (OSTRC questionnaire), oral health knowledge (8-item questionnaire), gingival inflammation, self-reported oral health problems and oral hygiene routines.

Results At baseline, 41 (66.1\%) athletes had evidence of caries, 29 (46.8\%) evidence of erosion, mean bleeding score was 11.57 (8.11), $44(71 \%)$ were male and 58 (93.5\%) white British. Mean OSTRC score reduced from 8.73 (14.54) to 2.73 (11.31) $\mathrm{p}<0.001$. Mean knowledge score improved from 5.69 (1.59) to $6.93(1.32) \mathrm{p}<0.001$. Gingival bleeding score was unchanged. Self-reported oral health problems reduced. Athlete use of prescription fluoride toothpaste $(2800 \mathrm{ppm})$ increased from $8(12.9 \%)$ to $45(80.4 \%, \mathrm{p}<0.001)$.

Conclusions This behaviour change theory-based intervention was implemented within different elite sport environments. It was associated with a reduction in self-reported performance impacts, an increase in athlete oral health knowledge and enhanced oral health behaviour.

\section{EYE INJURIES IN SPORTS: AN UPDATE}

Thomas Henke, Gernot Jendrusch, Petra Platen. Ruhr-University Bochum, Dep. of Sports Medicine, Bochum, Germany

\subsection{6/bjsports-2021-IOC.314}

Background About 3\% of all eye injuries happen during sports activities. About $25 \%$ of these eye injuries are so severe that they need ophthalmic treatment.

Objective Epidemiology of eye injuries in sports, risk of eye injuries in different sports.

Participants 2.392 eye injuries were analysed based on 221.273 sports injuries (club sports) that have been recorded by the sports injury database of the Ruhr-University Bochum and ARAG Sports Insurance between 1987 and 2017. In order to calculate the sports discipline related risk of eye injuries the frequency of eye injuries in one sports discipline has been qualified with regard to the frequency of all sports injuries that have been recorded in this sports discipline.

Results The average age of the 2.392 eye injured - this equals $1.08 \%$ of all sports injuries - was 31.9 (age range: 1 - 95 years). $78 \%$ of the injured persons were male, whereas $22 \%$ were female. About $19.7 \%$ had to stay in hospital, $13.9 \%$ needed surgery and $56.9 \%$ were incapable to work. On average, the injured had to pause their sport for 24.8 days. Blunt traumata, e.g. contusions, are dominant with over $50 \%$. These are often injuries caused by balls (e.g. in tennis or squash), rackets (e.g. in squash or ice hockey) or hand and elbow hits during tackles/tacklings (e.g. in handball or soccer). Assuming a mean risk of 1 for all sports, especially sports disciplines like squash (x8.4), badminton (x5.3), tennis (x4.8), but also water polo (x8.9) with swimming and grasping movements at eye level contain a significantly higher risk of eye injuries.

Conclusions Due to the special risk of eye injuries in squash the use of protective (sports) goggles is recommended from an 
ophthalmological point of view (already for a long time). For one-eyed athletes or sports persons with monocular defective vision protective sports goggles should be obligatory in order to protect the remaining healthy eye.

\section{HOW CAN WE PROTECT ATHLETES FROM DENTAL EROSION?}

${ }^{1}$ Hesham Matabdin, ${ }^{1}$ Paul Ashley, ${ }^{2}$ Pete Wilde, ${ }^{1}$ Ian Needleman. ${ }^{1}$ University College of London, Eastman Dental Institute, London, UK; ${ }^{2}$ Food structure, colloids and digestion, Quadram Institute, Norwich, UK

\subsection{6/bjsports-2021-IOC.315}

Background Our data on more than 800 participants shows that dental erosion (DE) affects up to $45 \%$ of elite athletes and may have physical and psychosocial impacts. The condition is associated with consumption of sports drinks and is exacerbated by reduced salivary flow. The protein rich layer called dental pellicle (DP) may have a protective function for $\mathrm{DE}$ and this might prove to be a useful therapeutic target in elite sport.

Objectives To assess the protective function of DP against DE. Design In vitro study.

Setting Laboratory.

Participants 40 bovine incisors

Interventions Two levels. 1: We exposed twenty bovine incisors to an energy drink ( $\mathrm{pH} 3.1)$ at 24,48 and 72 hours to create an in vitro erosion model. 2: We used fresh human saliva to form dental pellicle on the buccal surface of twenty incisors to create an in-vitro pellicle model. Erosive lesions were investigated both in the presence and absence of a layer of dental pellicle.

Main outcome measurement Mean depth of erosive lesions by optical coherence tomography (OCT) and X-ray Microtomography (XMT).

Results OCT showed the mean thickness of amorphous enamel before immersion in the erosive medium was $64 \mu \mathrm{m}$ (SD 2.3, IQR 54.7 - 63.2). The mean depth of the erosive lesion after immersion was $240.8 \mu \mathrm{m}$ (SD 32.7, IQR 244.6 - 263.8) $(p<0.001)$ at 72 hours. In contrast, mean depth of lesion in the presence of the dental pellicle was 84.3 (SD 18.9, IQR 93.2 - 75.3) $(p<0.03)$. XMT showed the mean depth of erosive lesions was $130 \mu \mathrm{m}$ (SD 28.2), whereas in the presence of DP was $82.4 \mu \mathrm{m}$ (SD 15.6) $(p<0.001)$.

Conclusions Erosion was reduced but not prevented by presence of dental pellicle. This model shows promise as a method to investigate novel interventions to prevent $\mathrm{DE}$ in elite athletes.

\section{MICROBIOME ANALYSIS IN ELITE SPORT}

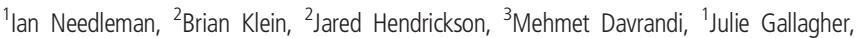
${ }^{1}$ Paul Ashley, ${ }^{3}$ David Spratt. 'UCL Eastman Dental Institute, Centre for Oral Health and Performance, London, UK; ${ }^{2}$ Vedanta Biosciences, Cambridge, USA; ${ }^{3}$ UCL Eastman Dental Institute, Microbial Diseases, London, UK

\subsection{6/bjsports-2021-IOC.316}

Background There is increasing interest in the microbiome in performance and prevention of illness.

Objective To investigate characteristics of oral and gut microbiomes in elite sport.
Design Metagenomic sequencing performed on stool and saliva samples at baseline and three months. Taxanomic identification of the DNA sequence data generated on the Illumina sequencing platform, followed by unsupervised Principle Component Analyses (PCA). UCL Research Ethics Committee ID Number: 6388/002.

Setting 1: A GB Olympic team, $\mathrm{n}=18 ; 2$ : English premiership rubgy team, $\mathrm{n}=18 ; 3$ : Healthy volunteers (non-athletes) $\mathrm{n}=28$. Participants Aged $\geq 18$ years, able to understand consent process, for health controls body mass index18 to $30 \mathrm{~kg} / \mathrm{m} 2$.

Assessment Of Risk Factors Oral health, BMI, use of antibiotics.

Outcome Measurements Unsupervised PCA.

Results Two distinct clusters emerged, one of athletes and one of non-athletes. Specific species-level signatures distiguishing the two clusters as well as each cohort were identified, including Fusicatenibacter sacchrivorans enriched in athlete samples relative to non-athlete controls, Slackia isoflavoniconvertans enriched in rugby relative to both Olympic athletes and nonathletes, and Klebsiella pnemoniae enriched in Olympic cohort relative to both rubgy and non-athletes (all $\mathrm{P}<0.005$ ). For saliva PCA analyses, no distinct clusters emerged between the two athlete cohorts or timepoints. However, specific specieslevel signatures distiguishing the groups were indentified, including multiple Neisseria spp. being elevated in rubgy relative to Olympic athetes at both timepoints, Prevotella histicola being elevated in Olympic athletes relative to rubgy at both timepoints, and Bifidobacterium longum being almost exclusively detected in the Olympic cohort but not rugby.

Conclusions Marked differences in microbiome signatures were found both between elite athletes and non-athletes and between team and individual sport cohorts. Further studies may help identify microbial factors related to optimal food conversion, performance or recovery, and prediction of illness risk.

\section{EQUIPMENT-RELATED RISK FACTORS FOR ACL INJURY AMONG RECREATIONAL SKIERS - A CASE CONTROL STUDY}

${ }^{1}$ Gerhard Ruedl, ${ }^{1,2}$ Markus Posch, ${ }^{2}$ Katja Tecklenburg, ${ }^{1}$ Martin Faulhaber ${ }^{1}$ Martin Burtscher. 'Department of Sport Science, University of Innsbruck, Innsbruck, Austria; ${ }^{2}$ Medalp sportclinic, Imst, Austria

\subsection{6/bjsports-2021-IOC.317}

Background In recreational skiing, an ACL injury is one of the most common diagnosis.

Objective To compare equipment-related risk factors between ACL injured and uninjured skiers.

Design Case-Control study over the 3 winter seasons 2016/ 17-2018/19 in one major Austrian ski resort.

Participants 248 ACL injured (52\% females) and 1054 uninjured skiers (48\% females).

Assessment of Risk Factors ACL injury was diagnosed by MRI in a sports clinic located in the ski area. Ski length [m] and side cut radius $[\mathrm{m}]$ were taken from the information given on the ski and ski length was relativized to body height [\%]. Combined height of ski and binding plate $[\mathrm{mm}]$ at the front $\left(\mathrm{H}_{\mathrm{F}}\right)$ and back part $\left(\mathrm{H}_{\mathrm{B}}\right)$ of the ski binding, and height at the toe $\left(\mathrm{H}_{\mathrm{T}}\right)$ and heel piece $\left(\mathrm{H}_{\mathrm{H}}\right)$ of the ski boot $[\mathrm{mm}]$ were measured with a digital sliding caliper. Quotients between $\mathrm{H}_{\mathrm{F}}$ and $\mathrm{H}_{\mathrm{B}}$ as well as between $\mathrm{H}_{\mathrm{T}}$ and $\mathrm{H}_{\mathrm{H}}$ were calculated. 